\title{
A Spontaneous Recurrent Seizure Bioassay for Anti-Epileptogenic Molecules
}

\author{
Angela P. Lyon, Dan Wainman, Sandra Marone, Donald F. Weaver
}

\begin{abstract}
Drug design in epilepsy is now tackling a new target - epileptogenesis. This is the process whereby a normal brain becomes susceptible to recurrent seizures. One of the stumbling blocks in the design and discovery of new chemical entities as antiepileptogenics is the implementation of an appropriate biological model. Current models, such as the maximal electroshock model, are models of seizures, not models of epileptogenesis. To develop such a model, we have extended and modified a chronic pilocarpine spontaneous recurrent seizure (SRS) model for the purposes of developing a bioassay with which to screen new compounds for putative antiepileptogenic bioactivity.
\end{abstract}

RÉSUMÉ: Un bioessai de crises convulsives récurrentes spontanées pour évaluer les molécules antiépileptogènes. La conception de médicaments antiépileptiques s'attaque maintenant à une nouvelle cible l'épileptogenèse, le processus par lequel un cerveau normal devient sujet à des crises épileptiques récurrentes. Une des pierres d'achoppement dans la conception et la découverte de nouveaux produits chimiques antiépileptogènes est la mise au point d'un modèle biologique approprié. Les modèles actuels comme le modèle d'électrochoc maximal sont des modèles de crises épileptiques et non pas des modèles d'épileptogenèse. Afin de développer un tel modèle, nous avons élargi et modifié un modèle de crises convulsives récurrentes spontanées en réponse à l'administration de pilocarpine dans le but de développer un bioessai pour évaluer l'activité antiépileptogène de nouveaux composés.

Can. J. Neurol. Sci. 2005; 32: 97-102

Epileptogenesis is a slow, insidious, biochemical and morphological process whereby normal brain is transformed (during a "latency period") into pathologically excitable neural tissue which is susceptible to spontaneous recurrent seizures. ${ }^{1,2}$ In humans, this process may culminate in a seizure disorder months to years following a brain injury. Traditional therapies for the treatment of epilepsy are unable to address epileptogenesis as a therapeutic goal. For example, various studies have failed to show antiepileptogenic effects for conventional anticonvulsant drugs, such as phenytoin or carbamazepine. ${ }^{3,4}$ Thus far there is no evidence that any current anticonvulsant drug administered during the latency period can prevent or even delay the development of epilepsy., 5 These currently available anticonvulsant medications do not target epileptogenesis, but rather inhibit ictogenesis - the rapidly propagating electrical and chemical event whereby aberrant electrical hyper-excitability spreads from an established seizure focus giving rise to the clinical manifestations of a single seizure. The market for anti-ictogenic (anticonvulsant, antiseizure) agents has been reasonably well-served over the past century with classical ion channel active agents such as phenytoin, carbamazepine and valproate and with newer generation medications such as lamotrigine. However, it is now a neuropharmacologic priority that the design strategy for antiepileptic molecules should extend beyond antiseizure agents to encompass the larger mandate of anti-epileptogenic agents.
Central to a comprehensive programme targeting the discovery of anti-epileptogenic agents is the need for an appropriate animal model. This model not only must accurately reflect the biology of epileptogenesis, but also must be useful for the facile screening of new chemical entities for potential bioactivity. Current widely used assays, such as the maximal electroshock (MES) model and the pentylenetetrazol (ScMet) model (and other related chemoconvulsant assays) are models of seizures, rather than models of epileptogenesis. Although kindling has been put forward as a model for epileptogenesis, the applicability of kindling as a procedure for identifying potential therapeutics for epilepsy has been debated. ${ }^{7,8}$ Accordingly, there is a continuing need for relevant models of epileptogenesis which can be used in the screening of new chemical entities.

In this paper, we describe an extension of the Spontaneous Recurrent Seizure (SRS) Model of Mello, Cavalheiro, et al. ${ }^{9}$ Models such as this, which employ pilocarpine or kainate

From the Department of Chemistry, Queen's University, Kingston, Ontario, (APL, DW, SM, DFW); Departments of Chemistry and Medicine (Neurology), School of Biomedical Engineering, Dalhousie University, Halifax, Nova Scotia, (DFW); Canada. ReCEIVED June 20, 2003. ACCEPTEDINFINALFORM July 27, 2004

Reprint requests to: D.F. Weaver, Departments of Chemistry and Medicine, Chemistry Building, Dalhousie University, Halifax, Nova Scotia, Canada, B3H 4J3 
induced chronic status epilepticus to ultimately induce a state of SRS, are now used to investigate the biology of epilepsy; ${ }^{10}$ however, they have not been used to screen unknown chemical compounds for potential anti-epileptogenic activity. We have modified the SRS model to enable evaluation of test compounds as potential anti-epileptogenic agents. Using a three step strategy, we describe the implementation, adaptation and integration of the SRS model into a comprehensive assay for screening new chemical entities as potential new antiepileptogenic drugs.

\section{Methods}

The central theme of this study concerns methodology development. Accordingly, the methods section is divided into three steps describing the implementation, adaptation and integration of the SRS model into an assay with which to screen molecules for possible anti-epileptogenic activity. All experiments involving animals had the approval of the Queen's University Animal Care Committee.

\section{Step 1: Implementation of the SRS model}

As a first step it was crucial that the SRS model be implemented in a manner that would enable accuracy and reproducibility so that it could be applied to large numbers of animals.

\section{The SRS model}

In refining the SRS model, initial "method refining" studies were performed using 40 rats with subsequent "method validation" studies on an additional 150 rats to ensure the accuracy and reproducibility of the model (15 groups of 10 rats). On Day 1, a group of 10 male Sprague-Dawley rats (175 -200 g, Charles River) was given methylscopolamine $(1 \mathrm{mg} / \mathrm{kg}$ intraperitoneally (i.p.)) to minimize the peripheral effects of pilocarpine (see Figure 1). Thirty minutes later, the animals were injected with pilocarpine $(380 \mathrm{mg} / \mathrm{kg}$ i.p., Aldrich Chemical Co.). Approximately 7-15 minutes later, $82 \%$ of the rats
$(123 / 150)$ entered a state of convulsive status epilepticus. In each group of 10 rats, $8.2 \pm 1.6$ (standard deviation) rats developed status epilepticus. Animals that did not undergo continuous seizure activity were not used any further in the study. Initially, the animals typically exhibited ear and whisker twitching, head jerking and body rearing with pedaling of the forepaws, followed by several minutes in which this behaviour diminished slightly; then the body movements started again and a constant seizure state was attained which persisted for hours. If the animal was able to sustain the continuous seizure activity while remaining on its feet (moderate severity) then the status epilepticus was allowed to last for three hours. However, if the seizure severity was severe and the animal was lying on its side, then the seizure was terminated after two hours. In our experience, animals that underwent a severe seizure for three hours did not survive the experiment. The mortality rate during the status epilepticus period was approximately $24 \%$. The seizure was stopped at the desired time (two hours for severe seizures; three hours for moderate severity) by the administration of diazepam $(2-4 \mathrm{mg} / \mathrm{kg}$ i.p.). This stopped the seizure activity after two minutes. Following the status epilepticus episode, the hydration level of the animals was supported by the administration of $20 \%$ sucrose solution (Gatorade ${ }^{\circledR}, 2.5 \mathrm{~mL}$, p.o.) and Ringer's lactate solution ( $5 \mathrm{~mL}$ i.p.) once a day for four days. Additionally, a portion of the rat chow pellets was ground into a paste using water. This soft food was available in the cage in a shallow dish. The animals were weighed each day. A $15-20 \%$ body weight loss was typical by day 2; normal weight gain was typically recovered after day 3.

Comments: [i] We experimented with varying doses of pilocarpine. Higher doses of pilocarpine $(>400 \mathrm{mg} / \mathrm{kg}$ ) produced unacceptably high mortality (>85\%); lower doses of pilocarpine produced unacceptably low rates of status epilepticus $(<50 \%)$. Therefore, the model was standardized with a pilocarpine dose of $380 \mathrm{mg} / \mathrm{kg}$. [ii] To ensure that the behavioural manifestations of the seizure corresponded with electroencephalographic (EEG) seizures, a subset of 10 rats had EEGs recorded during the status

Male Sprague-Dawley rat given methyl-scopolamine (1 mg/kg i.p.) and pilocarpine (380 mg/kg i.p.). Animal enters status epilepticus.

$$
\downarrow 2 \text { or } 3 \text { hours }
$$

Diazepam is administered (2-4 mg/kg i.p.). Seizure stops. Test compound injected 1/day for 7 days (20 mg/kg i.p.). Hydration support given during recovery.

$$
\downarrow 21 \text { days }
$$

Animals are placed in front of video cameras and videotaped 8 hours/day, 5 days/week for 2 months and then euthanised by sodium pentobarbital (100-120 mg/kg) \& cardiac puncture. Organs are examined histopathologically.

$\downarrow$

The number of seizures are determined from the videotapes viewed by individuals blinded to the test drug/control information.

Figure 1: Schematic of the SRS model for compound evaluation. 
epilepticus on day 1. A two channel EEG was recorded using jeweller's screws that had been inserted into the skull prior to the administration of methylscolpolamine. Although most rats showed appropriate EEG changes with the ear and whisker twitching, all rats showed evidence of generalized electrographic seizures concomitant with the body rearing and forepaw pedaling. Therefore, the behavioural seizures did correspond to electrographic seizures.

\section{Recovery and video-recording period}

The pilocarpine induced status epilepticus was induced on day 1 of the study period. Following the status epilepticus, the rats were placed individually in clear plexiglass cages with food and water provided via the top of the cage; wood shavings lined the bottom. Cages were changed daily; food and water were provided ad libitum. The light/dark cycle was maintained at $12 \mathrm{~h} / 12 \mathrm{~h}$. On day 21 , the animals were placed in front of video camera stations and were videotaped for eight hours/day (1000$1800 \mathrm{hrs}$ ), five days/week for eight weeks. Following two months of videotaping the animals were euthanized on day 81 .

Comments: [i] We experimented with varying the duration of video-taping (30 days vs. 60 days vs. 90 days). We found evidence of secondary epileptogenesis; that is, there were more seizures during month 2 (days 52-81) than in month 1 (days 2151) ("seizures beget seizures"). Thus, counting seizures for only one month was insufficient. On the other hand, counting seizures for three months added no additional information (other than larger numbers of seizures), and did add considerable time. Also, during the third month the behavioural severity of the seizures was often muted (i.e. pedaling forepaws without rearing) and became more difficult to detect. [ii] Rats were videotaped from 10:00-18:00 hrs during light hours. To ensure that this was the optimal recording timeframe, a subset of 10 rats was studied by being taped $24 \mathrm{hrs} /$ day (08:00-00:00 hrs [light]; 00:00-08:00 hrs [dim lighting]). There was a $24 \%$ reduction in seizure frequency during the dusk conditions, as compared to light conditions. Therefore, taping during the daylight hours afforded optimal timing for recording behavioural seizures.

\section{Postmortem examination of rats}

As part of the evaluation of a new chemical entity, it is desirable to evaluate the influence of the chemical upon brain histology. To permit appropriate neurohistopathological examination, the following protocol was followed. ${ }^{11}$ Two perfusion solutions were prepared: (1) $0.37 \%$ sulphide solution (pH 7.2; $11.7 \mathrm{~g} \mathrm{Na}_{2} \mathrm{~S} .9 \mathrm{H}_{2} \mathrm{O}$ plus $11.9 \mathrm{~g} \mathrm{NaH}_{2} \mathrm{PO}_{4} . \mathrm{H}_{2} \mathrm{O}$ made up to 1000 mLs. (2) $10 \%$ neutral buffered formalin (NBF; $90 \mathrm{~mL}$ of $37 \%$ formaldehyde, $3.6 \mathrm{~g} \mathrm{NaH} \mathrm{PO}_{4} \cdot \mathrm{H}_{2} \mathrm{O}$ and $5.85 \mathrm{~g} \mathrm{Na}_{2} \mathrm{HPO}_{4} .7 \mathrm{H}_{2} \mathrm{O}$ dissolved in $810 \mathrm{~mL}$ of distilled water. The animals were anaesthetized using sodium pentobarbital $(65 \mathrm{mg} / \mathrm{kg}$ i.p.) and the thoracic cavity was exposed. The descending aorta was tied off with a ligature. A needle containing the sulphide perfusion solution was then placed into the left ventricle and clamped into place. Another needle was rinsed with heparin (10000 U.S.P. units per $\mathrm{mL}$ ) and used to withdraw $5 \mathrm{~mL}$ of blood from the heart. Using scissors a nick was then placed in the right atrium. The $60 \mathrm{~mL}$ sulphide perfusion solution was flushed through followed immediately by $60 \mathrm{~mL}$ of neutral buffer formalin. The heart, lungs, liver, kidneys and brain were then removed. The brain was placed in a vial with neutral buffer formalin. The other organs were weighed and measured and a gross examination was performed. The blood was centrifuged and the plasma was stored at $-50^{\circ} \mathrm{C}$ to permit subsequent biochemical analyses.

Comments: [i] To ensure that the behavioural seizure manifestations of this model correspond with production of discernible central nervous system (CNS) pathology, a subset of 55 rats had postmortem brain histology examinations performed on cortical (frontal, parietal, temporal and occipital), subcortical and brainstem tissue. In accord with previous studies by Mello, Liu and others, ${ }^{12-15}$ the SRS model produced neuronal loss and damage in the hippocampus and related limbic structures, paralleling temporal lobe epilepsy. [ii] To ensure that the SRS model did not produce systemic non-CNS pathology which could be inappropriately ascribed to a candidate chemical compound undergoing screening (thereby limiting its promotion as a drug candidate), all animals that were videotaped had postmortem examinations of their organ systems. Pathological examination of heart, lungs, kidneys, liver, stomach, and intestines showed no significant evidence of pathology.

\section{Step 2: Adapting SRS model for compound screening}

To date, the SRS model has been used for studying the biology of epilepsy and has not been employed as an assay with which to identify a novel chemical compound with antiepileptogenic activity. Accordingly, the next step was to adapt the SRS model to permit test compound administration and evaluation with subsequent result interpretation.

\section{Administering test compounds}

To evaluate a compound as a potential anti-epileptogenic agent, a solution containing the test compound was administered five minutes after the cessation of the status epilepticus on day 1 . The test compound may be dissolved in either $0.9 \%$ aqueous saline or in solvent mixtures containing saline and dimethylsulfoxide (DMSO). We have employed solutions containing up to $50 \%$ DMSO. (Rats receiving vehicle only injections of 50/50 water/DMSO had the same number of seizures as rats receiving vehicle only injections with $0.9 \%$ saline.) The test compound was injected once a day for seven days $(20 \mathrm{mg} / \mathrm{kg}$ i.p.). Appropriate control groups were incorporated into this assay. For purposes of compound evaluation, the control group created by concomitantly administering vehicle solutions only (no test compound) to a subset of pilocarpine treated-status epilepticus rats was crucial in ascertaining compound efficacy.

Comments: [i] The timing of test compound administration on day 1 was selected to represent the human clinical condition. We have elected to administer test compounds at five minutes after the termination of the status epilepticus and thus at seven to eight minutes after the administration of diazepam. While arguably this approach is potentially flawed by the potential for drug-drug interactions between the diazepam and the test compound, it was selected to reflect the human situation in which a putative antiepileptogenic drug would be administered in the emergency room setting almost immediately following the administration of other agents, of which benzodiazepines are most widely used. Moreover, substantial delays in administering the test compound would limit its capacity to modulate ongoing postictal neurochemistry. [ii] Control studies showed that DMSO has no anti-epileptogenic effects even when employed at concentrations 
of up to $50 \%$. [iii] The administration of the test drug at $20 \mathrm{mg} / \mathrm{kg}$ once/day for seven days was meant to enable the administration of a reasonable dosage over an acceptable timeframe for identifying potential anti-epileptogenesis; clearly, a compound with activity would require dose-response studies with varying doses over varying timeframes of administration. The dose of 20 $\mathrm{mg} / \mathrm{kg}$ once/day for seven days are variables that must be further evaluated in future work. These variables will be crucial to the evaluation of new chemical entities as anti-epileptogenic agents. Time periods shorter than seven days may be sufficient; time periods longer than seven days may afford even greater protection. Starting the putative therapeutic later in the course of the epileptogenic process is also a variable that must be eventually considered. Even when these variables are optimized for this rat model, their generalizability to the human situation must likewise be optimized. [iv] Although it would enhance the likelihood of a compound demonstrating antiepileptogenic activity, at no time were test compounds administered prior to the induction of status epilepticus. Such a protocol would not reflect analogous human clinical conditions.

\section{Videotape viewing and training of videotape viewers}

Six cages were placed in each taping station with a contrasting dark background behind the cages. A digital clock was placed in the taping window. Each rat was assigned a randomly chosen six digit rat identification (ID) number. These numbers did not allow one test group to be discerned from another nor the control animals to be discernible from the other rats. Tags with the rat ID numbers printed in large font were posted on each cage. A typical camera station would have two test compounds and a control represented. The number of seizures were determined by individuals viewing the videotapes at a fast-forward reviewing speed. To ensure the required image clarity necessary for detecting seizures, video cassette recorders with four heads were used for the recording process. Seizures were defined as body rearing and forepaw pedaling with or without generalized motor involvement.

Undergraduate university students were easily trained to watch the videotapes on fast-forward. The one-day training process involved utilizing multiple training tapes. These training tapes had been prepared from a typical day of taping with several seizures recorded with more than one animal sustaining the seizures. A portion of the training tape was viewed displaying normal rat behaviour on both regular speed and fast-forward. The trainee was directed to continually circle their gaze over the cages and to observe the activity of each animal in turn. Several seizures were then viewed at regular speed and on fast-forward for training in recognizing the unusual posture and erect tail that frequently precede overt seizure activity. Lastly, the trainee was asked to watch two test tapes in which seizures were known to have been recorded; their observations were checked for accuracy. If any seizures were missed on the test tapes, the trainee had to repeat the training session. The first three tapes that a newly qualified viewer watched were also examined by an experienced viewer and the results were compared. Quality control and quality assurance methods were put in place to verify the seizure count data. Every tenth videotape produced was double-viewed to check the agreement between independent viewers. All videotape viewers were requested to view tapes on fast-forward and if unusual activity was identified to rewind and view this activity at regular speed.

Comment: We performed a number of trials to ascertain how many rats a viewer could monitor while still maintaining accuracy. The optimal number was four to six rats. Significant error rates (greater than $10 \%$ variability between two observers) occurred with less than four rats and more than six rats.

\section{Step 3: Integrating SRS model into a comprehensive screening program}

As a model for screening compounds for anti-epileptogenic activity, the SRS model has a significant limitation. The throughput in this model is very low. If one wishes to screen a collection of 200 molecules, the SRS model is too timedemanding and labour intensive. The effective integration of the SRS model into a comprehensive screening program necessitates the implementation of an initial prescreen which requires either in vitro assays (preferable) or high throughput in vivo assays.

The selection of an in vitro prescreen for the SRS model is rendered difficult by the lack of a fundamental understanding of the molecular basis of epileptogenesis. Although competitive receptor binding studies would constitute a time- and costeffective prescreen, the precise receptor population to be targeted in epileptogenesis remains unclear. Work by Sutula, ${ }^{16}$ Lothman $^{17}$ and others ${ }^{18,19}$ support the role of either or both of the $\gamma$-aminobutyric acid-A $\left(\mathrm{GABA}_{\mathrm{A}}\right)$ receptor and the N-methyl-D-aspartate (NMDA) receptor in the pathogenesis of epileptogenesis. The role of voltage gated ion channels, such as the sodium channel, in ictogenesis is well-appreciated and these transmembrane proteins may also influence epileptogenesis. Consequently, binding studies involving $\mathrm{GABA}_{\mathrm{A}}$, NMDA and/or $\mathrm{Na}^{+}$channel receptors would constitute a reasonable in vitro prescreen. The protocols of Falch et $\mathrm{al}^{20}$ and Baron et $\mathrm{al}^{21}$ are useful binding studies for the $\mathrm{GABA}_{\mathrm{A}}$ and NMDA receptors, respectively, and were incorporated into a comprehensive prescreen prior to the SRS model. It must be emphasized that the $\mathrm{GABA}_{\mathrm{A}}$, NMDA and/or $\mathrm{Na}^{+}$receptors may have absolutely nothing to do with the underlying molecular pathogenesis of epileptogenesis and, thus, their use as a prescreen may result in the inappropriate rejection of an effective antiepileptogenic molecule. However, given the time inefficiencies of the SRS model, some form of prescreen will ultimately be necessary for the practical implementation of the SRS bioasssay into an anti-epileptogenic drug screening program.

The selection of a high throughput in vivo screen is also difficult. Although faster than the SRS model, most in vivo screens are nevertheless slow and cumbersome. Like the SRS model, kindling is also a labour-intensive and time-protracted procedure performed over days. Thus, although it may reflect epileptogenesis, kindling is not an appropriate prescreen for SRS. Another approach to an in vivo prescreen would be to use animal models of seizures (e.g. maximal electroshock or pentelenetetrazole). For example, if a compound showed activity against chemoconvulsant induced seizures, then it could be evaluated for anti-epileptogenic activity. This approach is based upon the supposition that there is overlap between the mechanisms of ictogenesis and epileptogenesis. If receptors such as $\mathrm{GABA}_{\mathrm{A}}$, NMDA and the voltage-gated $\mathrm{Na}^{+}$channel are involved in epileptogenesis, then there is justification for such a 


\begin{abstract}
In vitro Tests $\rightarrow$ In vivo Anticonvulsant Tests $\rightarrow$ In vivo Antiepileptogenic Tests $\left(\mathrm{GABA}_{\mathrm{A}}, \mathrm{NMDA}\right) \quad$ (MES, ScMet) (SRS, Kindling)
\end{abstract}

Figure 2: A model testing sequence for identifying anti-epileptogenic compounds

prescreen, since these receptors clearly exert a role in ictogenesis. However, other molecules, such as nerve growth factor, ${ }^{22}$ do not play a role in ictogenesis, but may play a definite role in epileptogenesis. Molecules targeting such a receptor would be missed in an anti-ictogenic in vivo prescreen.

A potential comprehensive screening program which incorporates the SRS model following a prescreen is shown in Figure 2.

\section{Results AND Discussion}

Since Merritt and Putnam's historic discovery of phenytoin in the late 1930s, there have been many models put forth to screen novel chemicals as potential agents for the treatment of epilepsy (e.g. MES, ScMet, strychnine, bicuculline, picrotoxin, allylglycine, isoniazid, 3-mercaptoproprionate, beta-carboline, cobalt, penicillin, tungstic acid). Virtually all of these models have been models of seizures (ictogenesis) and not models of chronic epileptogenesis. Although models such as the MES and ScMet assays have been used extensively, they have been useful in finding anticonvulsants, not anti-epileptogenics. Since the need for anti-epileptogenics is emerging as a neuropharmacologic priority, the need for an appropriate animal model with which to discover anti-epileptogenics is also a priority.

Whether kindling is such an animal model is open to debate. As an experimental technique, kindling has been available for many years; however, it has failed to yield an anti-epileptogenic drug (perhaps because no effective anti-epileptogenic agent has yet been evaluated in the kindling model). Kindling refers to the process whereby a subthreshold stimulus (usually a focal electrical stimulation of a limbic or cortical structure) repeated over days or weeks becomes a convulsant stimulus. ${ }^{23}$ Kindling is a relatively time consuming procedure as it requires the chronic implantation of stimulating electrodes in the amygdala, hippocampus, or cortex, and daily electrical stimulation with scoring of either the after-discharge duration or of the motor seizure response. Furthermore, because the relationship between kindling and spontaneous human epilepsy remains controversial, kindling is not considered to be an ideal model of epileptogenesis. ${ }^{24}$ Another criticism of the kindling model as an assay with which to identify a potential antiepileptogenic compound is that the test substance is administered before the kindling stimulation. Finally, even though a drug might be found to retard the kindling process, the fully kindled rat does not normally exhibit spontaneous seizures; therefore, it is impossible to conclude whether the ability of the drug to block kindling will inhibit the fundamental mechanistic processes of epileptogenesis. ${ }^{24}$ Clearly, there exists a need for an animal model which reflects the biology of epileptogenesis and which may be used to evaluate candidate molecules as anti-epileptogenics. Such a model may permit the identification of an anti-epileptogenic which is as useful as phenytoin has been as an anticonvulsant.

The adaptation of the SRS model of epileptogenesis as presented in this study is a model that strives to represent the human situation in which an anti-epileptogenic medication would be given following a head injury. The SRS model has biological and clinical relevance. Although the SRS model is biological relevant, the time requirements and the large amount of labour involved in the videotape viewing is a drawback of this screening program. In addition, the SRS model tends to produce obvious (even dramatic) brain pathology that may not accurately reflect the somewhat more subtle neuropathology of human epileptogenesis. The SRS bioassay was developed in rats and there may be species differences that limit its generalizability to the corresponding human situation. Nevertheless, this assay does provide a model of epileptogenesis rather than merely a model of seizures. Moreover, with the modifications described in this paper (including the use of in vitro or in vivo prescreens to facilitate efficiency and throughput), the SRS model may be used to evaluate novel compounds.

\section{ACKNOWLeDGeMents}

This work is supported in part by operating grants from the Canadian Institutes of Health Research, and the Natural Sciences and Engineering Research Council of Canada. DFWis a Tier 1 Canada Research Chair in Neuroscience. None of the four authors declares any conflict of interest in the contents of this manuscript.

\section{REFERENCES}

1. Lothman EW. Neurobiology as a basis for rational polypharmacy. Epilepsy Res 1996; (Suppl 11): 3-7.

2. Weaver DF. Epileptogenesis, ictogenesis and the design of future antiepileptic drugs. Can J Neurol Sci 2003; 30: 4-7.

3. Young B, Rapp RP, Norton JA, et al. Failure of prophylactically administered phenytoin to prevent late posttraumatic seizures. J Neurosurg 1983; 58(2): 236-241.

4. Glotzner FL, Haubitz I, Miltner F, Kapp G, Pflughaupt KW. Seizure prevention using carbamazepine following severe brain injuries. Neurochirurgia 1983; 26(3): 66-79.

5. Pitkanen A. Drug-mediated neuroprotection and antiepileptogenesis. Neurology 2002; 59 (Suppl 5): S27-S33.

6. Pitkanen A. Efficacy of current antiepileptics to prevent neurodegeneration in epilepsy models. Epilepsy Res 2002; 50: 141-160.

7. Girgis M. Kindling as a model of limbic epilepsy. Neuroscience 1981; 6: 1695-1706.

8. Goldensohn ES. The relevance of secondary epileptogenesis to the treatment of epilepsy: kindling and the mirror focus. Epilepsia 1984; 25 (Supp1 2): S156-S168.

9. Mello LEAM, Cavalheiro EA, Tan AM, et al. Molecular neurobiology of epilepsy. Epilepsy Res 1992; (Suppl 9): 51-60. 
10. Lothman EW. The biochemical basis and pathophysiology of status epilepticus. Neurology 1990; 40 (Suppl 2): 13-23.

11. Sloviter RS. A simplified Timm stain procedure compatible with formaldehyde fixation and routine paraffin embedding of rat brain. Brain Res Bull 1982; 8: 771-774.

12. Mello LEAM, Cavalheiro EA, Tan AM, et al. Circuit mechanisms of seizures in the pilocarpine model of chronic epilepsy: cell loss and mossy fibre sprouting. Epilepsia 1993; 34(6): 985-995.

13. Liu Z, Nagaro T, Desjardins GC, Gloor P, Avoli M. Quantitative evaluation of neuronal loss in the dorsal hippocampus of rats with long-term pilocarpine seizures. Epilepsy Res 1994; 17: 237-247.

14. Poirier JL, Capek R, De Koninck Y. Differential progression of dark neuron and fluoro-jade labeling in the rat hippocampus following pilocarpine-induced status epilepticus. Neuroscience 2000; 97(1): 59-68.

15. Coulter DA, Rafiq A, Shumate $M$, et al. Brain injury-induced enhanced limbic epileptogenesis: anatomical and physiological parallels to an animal model of temporal lobe epilepsy. Epilepsy Res 1996; 26: 81-91.

16. Lynch M, Sayin U, Golarai G, Sutula T. NMDAreceptor-dependent plasticity of granule cell spiking in the dentate gyrus of normal and epileptic rats. J Neurophysiol 2000; 84(6): 2868-2879.

17. Bekenstein JW, Lothman EW. Dormancy of inhibitory interneurons in a model of temporal lobe epilepsy. Science 1993; 259(5091): $97-100$.

18. Houser AR, Esclapez M. Vulnerability and plasticity of the GABA system in the pilocarpine model of spontaneous recurrent seizures. Epilepsy Res 1996; 26: 207-218.

19. Rice AC, DeLorenzo RJ. NMDA receptor activation during status epilepticus is required for the development of epilepsy. Brain Res 1998; 782: 240-247.

20. Sloviter R. Permanently altered hippocampal structure, excitability, and inhibition after experimental status epilepticus in the rat: the dormant basket cell hypothesis and its possible relevance to temporal lobe epilepsy. Hippocampus 1991; 1: 41-66.

20. Falch E, Hedegaard A. Competitive stereostructure-activity studies on GABA and $\mathrm{GABA}_{\mathrm{B}}$ receptor sites and GABA uptake using rat brain membrane preparations. J Neurochem 1986; 47(3): 898903.

21. Baron BM, Harrison BL, Kehne JH, et al. Pharmacological characterization of MDL-105.519 an NMDAreceptor glycine site antagonist. Eur J Pharmacol 1997; 323: 181-192.

22. Adams B, Sazgar M, Osehobo P, et al. Nerve growth factor accelerates seizure development, enhances mossy fiber sprouting, and attenuates seizure-induced decreases in neuronal density in the kindling model of epilepsy. J Neurosci 1997; 17(14): 52885296.

23. Racine RJ. Modification of seizure activity by electrical stimulation. ii. motor seizure. Electroencephalogr Clin Neurophysiol 1972; 32: 281-294.

24. White HS. Animal models of epileptogenesis. Neurology 2002; 59 (Suppl 5): S7-S14. 